Mathematical Modelling AND ANALysis

Volume 18 Number 1, February 2013, 149-159

http://dx.doi.org/10.3846/13926292.2013.760115

(c) Vilnius Gediminas Technical University, 2013
Publisher: Taylor\&Francis and VGTU

http://www.tandfonline.com/TMMA

Print ISSN: 1392-6292

Online ISSN: 1648-3510

\title{
A Joint Limit Theorem for Periodic Hurwitz Zeta-Functions with Algebraic Irrational Parameters
}

\author{
Danutè Geniene் $\dot{\mathrm{e}}^{a}$ and Audronè Rimkevičien $\dot{\mathrm{e}}^{b}$ \\ ${ }^{a}$ Šiauliai University \\ P. Višinskio 25, LT-77156 Šiauliai, Lithuania \\ ${ }^{b}$ Šiauliai State College \\ Aušros ave. 40, LT-76241 Šiauliai, Lithuania \\ E-mail(corresp.): dana@splius.lt \\ E-mail: audronerim@gmail.com
}

Received June 21, 2012; revised October 21, 2012; published online February 1, 2013

\begin{abstract}
In the paper, a joint limit theorem for weakly convergent probability measures in $\mathbb{C}^{r}$ for periodic Hurwitz zeta-functions with algebraic irrational parameters satisfying certain independence conditions is obtained.
\end{abstract}

Keywords: Hurwitz zeta-function, joint limit theorem, probability measure, weak convergence.

AMS Subject Classification: 11M41.

\section{Introduction}

Let $\mathfrak{a}=\left\{a_{m}: m \in \mathbb{N}_{0}=\mathbb{N} \cup\{0\}\right\}$ be a periodic sequence of complex numbers with minimal period $k \in \mathbb{N}$, and let $\alpha, 0<\alpha \leq 1$, be a fixed parameter. The periodic Hurwitz zeta-function $\zeta(s, \alpha ; \mathfrak{a}), s=\sigma+\mathrm{i} t$, is defined, for $\sigma>1$, by the series

$$
\zeta(s, \alpha ; \mathfrak{a})=\sum_{m=0}^{\infty} \frac{a_{m}}{(m+\alpha)^{s}},
$$

and is meromorphically continued to the whole complex plane by using the equality

$$
\zeta(s, \alpha ; \mathfrak{a})=\frac{1}{k^{s}} \sum_{l=0}^{k-1} a_{l} \zeta\left(s, \frac{\alpha+l}{k}\right),
$$

where $\zeta(s, \alpha)$ denotes the classical Hurwitz zeta-function. The point $s=1$ is the unique possible simple pole of $\zeta(s, \alpha ; \mathfrak{a})$.

In [6], the second author began to characterize the asymptotic behaviour of the function $\zeta(s, \alpha ; \mathfrak{a})$ by limit theorems on the weak convergence of probability 
measures. We discussed the cases of transcendental, rational and algebraic irrational parameter $\alpha$. The simplest of them is the case of transcendental $\alpha$ because of the linear independence over the field of rational numbers $\mathbb{Q}$ of the set $L(\alpha)=\left\{\log (m+\alpha): m \in \mathbb{N}_{0}\right\}$. The case of rational $\alpha$ is based on the linear independence over $\mathbb{Q}$ of the set $\{\log p: p \in \mathcal{P}\}$, where $\mathcal{P}$ denotes the set of all prime numbers. The most complicated case is that of algebraic irrational $\alpha$. In this case, there is no precise information on the linear independence of the set $L(\alpha)$. We know only a very deep theorem of Cassels which asserts that at least 51 percent of elements of the set $L(\alpha)$ in the sense of density are linear independent over $\mathbb{Q}$. The latter theorem allows to prove a limit theorem for weakly convergent probability measures with explicitly given limit measure.

The paper [7] is devoted to joint limit theorems for periodic Hurwitz zetafunctions. Let $\zeta\left(s, \alpha_{1} ; \mathfrak{a}_{1}\right), \ldots, \zeta\left(s, \alpha_{r} ; \mathfrak{a}_{r}\right)$ be a collection of periodic Hurwitz zeta-functions. In [7], two cases of the parameters $\alpha_{1}, \ldots, \alpha_{r}$ were discussed. The first case is of algebraically independent $\alpha_{1}, \ldots, \alpha_{r}$. Let $\gamma=\{s \in \mathbb{C}$ : $|s|=1\}$ be the unit circle on the complex plane, and $\Omega_{1}=\prod_{m=0}^{\infty} \gamma_{m}$, where $\gamma_{m}=\gamma$ for all $m \in \mathbb{N}_{0}$. Then $\Omega_{1}$ is a compact topological group. Define

$$
\underline{\Omega}_{1}=\prod_{j=1}^{r} \Omega_{1 j},
$$

were $\Omega_{1 j}=\Omega_{1}$ for $j=1, \ldots, r$. Then $\underline{\Omega}_{1}$ is also a compact topological group. Therefore, on $\left(\underline{\Omega}_{1}, \mathcal{B}\left(\underline{\Omega}_{1}\right)\right)(\mathcal{B}(S)$ denotes the class of Borel sets of the space $S)$ the probability Haar measure $\underline{m}_{1 H}$ exists, and we obtain the probability space $\left(\underline{\Omega}_{1}, \mathcal{B}\left(\underline{\Omega}_{1}\right), \underline{m}_{1 H}\right)$. Denote by $\underline{\omega}_{1}=\left(\omega_{11}, \ldots, \omega_{1 r}\right)$ the elements of the group $\underline{\Omega}_{1}$, and, on the probability space $\left(\underline{\Omega}_{1}, \mathcal{B}\left(\underline{\Omega}_{1}\right), \underline{m}_{1 H}\right)$, define $\mathbb{C}^{r}$-valued random element $\underline{\zeta}_{1}\left(\underline{\sigma}, \underline{\alpha}, \underline{\omega}_{1} ; \underline{\mathfrak{a}}\right)$ by the formula

$$
\underline{\zeta}_{1}\left(\underline{\sigma}, \underline{\alpha}, \underline{\omega}_{1} ; \underline{\mathfrak{a}}\right)=\left(\zeta_{1}\left(\sigma_{1}, \alpha_{1}, \omega_{11} ; \mathfrak{a}_{1}\right), \ldots, \zeta_{1}\left(\sigma_{r}, \alpha_{r}, \omega_{1 r} ; \mathfrak{a}_{r}\right)\right),
$$

where $\underline{\sigma}=\left(\sigma_{1}, \ldots, \sigma_{r}\right), \underline{\alpha}=\left(\alpha_{1}, \ldots, \alpha_{r}\right)$ and $\underline{\mathfrak{a}}=\left(\mathfrak{a}_{1}, \ldots, \mathfrak{a}_{r}\right)$, and

$$
\zeta_{1}\left(\sigma_{j}, \alpha_{j}, \omega_{1 j} ; \mathfrak{a}_{j}\right)=\sum_{m=0}^{\infty} \frac{a_{m j} \omega_{1 j}(m)}{\left(m+\alpha_{j}\right)^{\sigma_{j}}}, \quad \sigma_{j}>\frac{1}{2}, j=1, \ldots, r .
$$

Here $\mathfrak{a}_{j}=\left\{a_{m j}: m \in \mathbb{N}_{0}\right\}, j=1, \ldots, r$, are periodic sequences of complex numbers, and $\omega_{1 j}(m)$ denotes the projection of $\omega_{1 j} \in \Omega_{1 j}$ to the coordinate space $\gamma_{m}$. Let $P_{\underline{\zeta}_{1}}$ be the distribution of the random element $\underline{\zeta}_{1}\left(\underline{\sigma}, \underline{\alpha}, \underline{\omega}_{1} ; \underline{\mathfrak{a}}\right)$ and $\zeta(\underline{\sigma}+\mathrm{i} t, \underline{\alpha} ; \underline{\mathfrak{a}})=\left(\zeta\left(\sigma_{1}+\mathrm{i} t_{1}, \alpha_{1} ; \mathfrak{a}_{1}\right), \ldots, \zeta\left(\sigma_{r}+\mathrm{i} t_{r}, \alpha_{r} ; \mathfrak{a}_{r}\right)\right)$. Then the first theorem of [7] is the following statement.

Theorem 1. Suppose that $\min _{1 \leq j \leq r} \sigma_{j}>\frac{1}{2}$, and that the numbers $\alpha_{1}, \ldots, \alpha_{r}$ are algebraically independent over $\mathbb{Q}$. Then

$$
\frac{1}{T} \operatorname{meas}\{t \in[0, T]: \underline{\zeta}(\underline{\sigma}+\mathrm{i} t, \underline{\alpha} ; \underline{\mathfrak{a}}) \in A\}, \quad A \in \mathcal{B}\left(\mathbb{C}^{r}\right),
$$

converges weakly to $P_{\underline{\zeta}_{1}}$ as $T \rightarrow \infty$. 
Here and in the sequel, meas $\{\mathrm{A}\}$ stands for the Lebesque measure of a measurable set $A \subset \mathbb{R}$. The second joint limit theorem of [7] deals with rational parameters $\alpha_{1}, \ldots, \alpha_{r}$. In this case, we use $\Omega_{2}=\prod_{p \in \mathcal{P}} \gamma_{p}$, where $\gamma_{p}=\gamma$ for all primes $p$. The torus $\Omega_{2}$ is also a compact topological group, and we obtain a new probability space $\left(\Omega_{2}, \mathcal{B}\left(\Omega_{2}\right), m_{2 H}\right)$, where $m_{2 H}$ is the probability Haar measure on $\left(\Omega_{2}, \mathcal{B}\left(\Omega_{2}\right)\right)$. Denote by $\omega_{2}(p)$ the projection of $\omega_{2} \in \Omega_{2}$ to the coordinate space $\gamma_{p}, p \in \mathbb{P}$, and extend the function $\omega_{2}(p)$ to the set $\mathbb{N}$ by the formula

$$
\omega_{2}(m)=\prod_{p^{l} \| m} \omega_{2}^{l}(p), \quad m \in \mathbb{N} .
$$

Suppose that $\alpha_{j}=a_{j} / q_{j}, 0<a_{j}<q_{j},\left(a_{j}, q_{j}\right)=1, j=1, \ldots, r$, and, on the probability space $\left(\Omega_{2}, \mathcal{B}\left(\Omega_{2}\right), m_{2 H}\right)$, define the $\mathbb{C}^{r}$-valued random element $\underline{\zeta}_{2}\left(\underline{\sigma}, \underline{\alpha}, \omega_{2} ; \underline{\mathfrak{a}}\right)$ by the formula

$$
\underline{\zeta}_{2}\left(\underline{\sigma}, \underline{\alpha}, \omega_{2} ; \underline{\mathfrak{a}}\right)=\left(\zeta_{2}\left(\sigma_{1}, \alpha_{1}, \omega_{2} ; \mathfrak{a}_{1}\right), \ldots, \zeta_{2}\left(\sigma_{r}, \alpha_{r}, \omega_{2} ; \mathfrak{a}_{r}\right)\right),
$$

where, for $\sigma_{j}>\frac{1}{2}$,

$$
\zeta_{2}\left(\sigma_{j}, \alpha_{j}, \omega_{2} ; \mathfrak{a}_{j}\right)=\omega_{2}\left(q_{j}\right) q_{j}^{\sigma_{j}} \sum_{\substack{m=1 \\ m \equiv a_{j}\left(\bmod q_{j}\right)}}^{\infty} \frac{a_{\left(m-a_{j}\right) / q_{j}} \omega_{2}(m)}{m^{\sigma_{j}}}, j=1, \ldots, r .
$$

Let $P_{\underline{\zeta}_{2}}$ be the distribution of the random element $\underline{\zeta}_{2}\left(\underline{\sigma}, \underline{\alpha}, \omega_{2} ; \underline{\mathfrak{a}}\right)$. Then the second theorem of [7] is of the form.

Theorem 2. For $j=1, \ldots, r$, suppose that $\alpha_{j}=\frac{a_{j}}{q_{j}}, 0<a_{j}<q_{j},\left(a_{j}, q_{j}\right)=1$, and that $\sigma_{j}>\frac{1}{2}$. Then

$$
\frac{1}{T} \operatorname{meas}\{t \in[0, T]: \underline{\zeta}(\underline{\sigma}+\mathrm{i} t, \underline{\alpha} ; \underline{\mathfrak{a}}) \in A\}, \quad A \in \mathcal{B}\left(\mathbb{C}^{r}\right),
$$

converges weakly to $P_{\underline{\zeta}_{2}}$ as $T \rightarrow \infty$.

The aim of this paper is to obtain a joint limit theorem for periodic Hurwitz zeta-functions with algebraic irrational parameters. The motivation for this is a possible application of limit theorems in the investigation of the universality for periodic Hurwitz zeta-functions.

For $j=1, \ldots, r$, let $I\left(\alpha_{j}\right)$ be a maximal linearly independent over $\mathbb{Q}$ subset of the set $L\left(\alpha_{j}\right)$. Suppose that $L\left(\alpha_{j}\right) \neq I\left(\alpha_{j}\right)$, and define $D\left(\alpha_{j}\right)=L\left(\alpha_{j}\right) \backslash$ $I\left(\alpha_{j}\right)$. If $d_{j m}=\log \left(m+\alpha_{j}\right) \in D\left(\alpha_{j}\right)$, then the set $I\left(\alpha_{j}\right) \cup\left\{d_{j m}\right\}$ is already linearly dependent over $\mathbb{Q}$. Thus, there exist elements $i_{j m_{1}}, \ldots, i_{j m_{n}} \in I\left(\alpha_{j}\right)$ and $k_{j 0}, \ldots, k_{j n} \in \mathbb{Z} \backslash\{0\}$ such that

$$
d_{j m}=-\frac{k_{j 1}}{k_{j 0}} i_{j m_{1}}-\cdots-\frac{k_{j n}}{k_{j 0}} i_{j m_{n}} .
$$

From this we find that

$$
m+\alpha_{j}=\left(m_{1}+\alpha_{j}\right)^{-\frac{k_{j 1}}{k_{j 0}}} \cdots\left(m_{n}+\alpha_{j}\right)^{-\frac{k_{j n}}{k_{j 0}}} .
$$


Define the sets

$$
\mathcal{M}\left(\alpha_{j}\right)=\left\{m \in \mathbb{N}_{0}: \log \left(m+\alpha_{j}\right) \in I\left(\alpha_{j}\right)\right\}
$$

and

$$
\mathcal{N}\left(\alpha_{j}\right)=\left\{m \in \mathbb{N}_{0}: \log \left(m+\alpha_{j}\right) \in D\left(\alpha_{j}\right)\right\}
$$

Now let

$$
\Omega_{3 j}=\prod_{m \in \mathcal{M}\left(\alpha_{j}\right)} \gamma_{m}
$$

where $\gamma_{m}=\gamma$ for all $m \in \mathcal{M}\left(\alpha_{j}\right)$, and $\underline{\Omega}_{3}=\prod_{j=1}^{r} \Omega_{3 j}$. Then $\underline{\Omega}_{3}$ is a compact topological Abelian group. Therefore, on $\left(\underline{\Omega}_{3}, \mathcal{B}\left(\underline{\Omega}_{3}\right)\right)$, the probability Haar measure $\underline{m}_{3 H}$ can be defined, and we obtain the probability space $\left(\underline{\Omega}_{3}, \mathcal{B}\left(\underline{\Omega}_{3}\right), \underline{m}_{3 H}\right)$. Denote by $\omega_{3 j}(m)$ the projection of $\omega_{3 j} \in \Omega_{3 j}$ to $\gamma_{m}$, $m \in \mathcal{M}\left(\alpha_{j}\right)$, and extend the function $\omega_{3 j}(m)$ to the set $\mathbb{N}_{0}$ by the formula

$$
\omega_{3 j}(m)=\omega_{3 j}\left(m_{1}\right)^{-\frac{k_{j 1}}{k_{j 0}}} \cdots \omega_{3 j}\left(m_{n}\right)^{-\frac{k_{j n}}{k_{j 0}}}, \quad m \in \mathcal{N}\left(\alpha_{j}\right)
$$

if equality (1.1) holds. Here the principal values of roots are taken. Denote by $\underline{\omega}_{3}=\left(\omega_{31}, \ldots, \omega_{3 r}\right)$ the elements of the group $\underline{\Omega}_{3}$, and, on the probability space $\left(\underline{\Omega}_{3}, \mathcal{B}\left(\underline{\Omega}_{3}\right), \underline{m}_{3 H}\right)$, define the $\mathbb{C}^{r}$-valued random element $\underline{\zeta}_{3}\left(\underline{\sigma}, \underline{\alpha}, \underline{\omega}_{3} ; \underline{\mathfrak{a}}\right)$ by the formula

$$
\underline{\zeta}_{3}\left(\underline{\sigma}, \underline{\alpha}, \underline{\omega}_{3} ; \underline{\mathfrak{a}}\right)=\left(\zeta_{3}\left(\sigma_{1}, \alpha_{1}, \omega_{31} ; \mathfrak{a}_{1}\right), \ldots, \zeta_{3}\left(\sigma_{r}, \alpha_{r}, \omega_{3 r} ; \mathfrak{a}_{r}\right)\right),
$$

where, for $\sigma_{j}>\frac{1}{2}$,

$$
\zeta_{3}\left(\sigma_{j}, \alpha_{j}, \omega_{3 j} ; \mathfrak{a}_{j}\right)=\sum_{m=0}^{\infty} \frac{a_{m j} \omega_{3 j}(m)}{\left(m+\alpha_{j}\right)^{\sigma_{j}}}, \quad j=1, \ldots, r .
$$

Denote by $P_{\underline{\zeta}_{3}}$ the distribution of the random element $\underline{\zeta}_{3}\left(\underline{\sigma}, \underline{\alpha}, \underline{\omega}_{3} ; \underline{\mathfrak{a}}\right)$. The main result of the paper is the following theorem.

Theorem 3. Suppose that the numbers $\alpha_{1}, \ldots, \alpha_{r}$ are algebraic irrational, the set $\bigcup_{j=1}^{r} I\left(\alpha_{j}\right)$ is linearly independent over $\mathbb{Q}$, and that $\min _{1 \leq j \leq r} \sigma_{j}>\frac{1}{2}$. Then

$$
P_{T}(A) \stackrel{\text { def }}{=} \frac{1}{T} \operatorname{meas}\{t \in[0, T]: \underline{\zeta}(\underline{\sigma}+\mathrm{i} t, \underline{\alpha} ; \underline{\mathfrak{a}}) \in A\}, \quad A \in \mathcal{B}\left(\mathbb{C}^{r}\right),
$$

converges weakly to $P_{\underline{\zeta}_{3}}$ as $T \rightarrow \infty$.

Theorem 3 is the first attempt to obtain probabilistic limit theorems used in proofs of universality theorems for zeta-functions. On the other hand, Theorem 3 characterizes the asymptotic behaviour of a collection of periodic Hurwitz zeta-functions with algebraic irrational parameters. This is a motivation of the paper. 


\section{A Limit Theorem on the Torus $\underline{\Omega}_{3}$}

In this section, we consider the weak convergence of

$$
\begin{aligned}
Q_{T}(A)= & \frac{1}{T} \operatorname{meas}\left\{t \in[0, T]:\left(\left(\left(m+\alpha_{1}\right)^{-\mathrm{i} t}: m \in \mathcal{M}\left(\alpha_{1}\right)\right), \ldots,\right.\right. \\
& \left.\left.\left(\left(m+\alpha_{r}\right)^{-\mathrm{i} t}: m \in \mathcal{M}\left(\alpha_{r}\right)\right)\right) \in A\right\}, \quad A \in \mathcal{B}\left(\underline{\Omega}_{3}\right) .
\end{aligned}
$$

Theorem 4. Suppose that the numbers $\alpha_{1}, \ldots, \alpha_{r}$ satisfy the hypotheses of Theorem 3. Then $Q_{T}$ converges weakly to the Haar measure $\underline{m}_{3 H}$ as $T \rightarrow \infty$.

Proof. The dual group of $\underline{\Omega}_{3}$ is isomorphic to

$$
\mathcal{D} \stackrel{\text { def }}{=}\left(\bigoplus_{m \in \mathcal{M}\left(\alpha_{1}\right)} \mathbb{Z}_{m}\right) \oplus \cdots \oplus\left(\bigoplus_{m \in \mathcal{M}\left(\alpha_{r}\right)} \mathbb{Z}_{m}\right)
$$

where $\mathbb{Z}_{m}=\mathbb{Z}$ for all $m \in \mathcal{M}\left(\alpha_{j}\right), j=1, \ldots, r$. An element $\underline{k}=\left(k_{1 m}: m \in\right.$ $\left.\mathcal{M}\left(\alpha_{1}\right), \ldots, k_{r m}: m \in \mathcal{M}\left(\alpha_{r}\right)\right)$ of $\mathcal{D}$, where only a finite number of integers $k_{j m}, j=1, \ldots, r$, are distinct from zero, acts on $\underline{\Omega}_{3}$ by

$$
\underline{x} \rightarrow \underline{x}^{\underline{k}}=\prod_{m \in \mathcal{M}\left(\alpha_{1}\right)} x_{1 m}^{k_{1 m}} \cdots \prod_{m \in \mathcal{M}\left(\alpha_{r}\right)} x_{r m}^{k_{r m}},
$$

where $\underline{x}=\left(\left(x_{1 m}: m \in \mathcal{M}\left(\alpha_{1}\right)\right), \ldots,\left(x_{r m}: m \in \mathcal{M}\left(\alpha_{r}\right)\right)\right) \in \underline{\Omega}_{3}$. Therefore, the Fourier transform $g_{T}(\underline{k})$ of the measure $Q_{T}$ is of the form

$$
\begin{aligned}
g_{T}(\underline{k})= & \int_{\underline{\Omega}_{3}}\left(\prod_{m \in \mathcal{M}\left(\alpha_{1}\right)} x_{1 m}^{k_{1 m}} \ldots \prod_{m \in \mathcal{M}\left(\alpha_{r}\right)} x_{r m}^{k_{r m}}\right) \mathrm{d} Q_{T} \\
= & \frac{1}{T} \int_{0}^{T}\left(\prod_{m \in \mathcal{M}\left(\alpha_{1}\right)}\left(m+\alpha_{1}\right)^{-\mathrm{i} t k_{1 m}} \ldots \prod_{m \in \mathcal{M}\left(\alpha_{r}\right)}\left(m+\alpha_{r}\right)^{-\mathrm{i} t k_{r m}}\right) \mathrm{d} t \\
= & \frac{1}{T} \int_{0}^{T} \exp \left\{-\mathrm{i} t\left(\sum_{m \in \mathcal{M}\left(\alpha_{1}\right)} k_{1 m} \log \left(m+\alpha_{1}\right)+\cdots\right.\right. \\
& \left.\left.+\sum_{m \in \mathcal{M}\left(\alpha_{r}\right)} k_{r m} \log \left(m+\alpha_{r}\right)\right)\right\} \mathrm{d} t
\end{aligned}
$$

where only a finite number of integers $k_{j m}, j=1, \ldots, r$, are distinct from zero. Since the set $\bigcup_{j=1}^{r} I\left(\alpha_{j}\right)$ is linearly independent over $\mathbb{Q}$, we have that

$$
l(\underline{k}) \stackrel{\text { def }}{=} \sum_{m \in \mathcal{M}\left(\alpha_{1}\right)} k_{1 m} \log \left(m+\alpha_{1}\right)+\cdots+\sum_{m \in \mathcal{M}\left(\alpha_{r}\right)} k_{r m} \log \left(m+\alpha_{r}\right)=0
$$

if and only if $\underline{k}=\underline{0}$. Therefore, after integration in (2.1), we find that

$$
g_{T}(\underline{k})= \begin{cases}1 & \text { if } \underline{k}=\underline{0} \\ \frac{\exp \{-\mathrm{i} T l(\underline{k})\}-1}{-\mathrm{i} T l(\underline{k})} & \text { if } \underline{k} \neq \underline{0} .\end{cases}
$$


Thus,

$$
\lim _{T \rightarrow \infty} g_{T}(\underline{k})= \begin{cases}1 & \text { if } \underline{k}=\underline{0} \\ 0 & \text { if } \underline{k} \neq \underline{0}\end{cases}
$$

and it follows from a continuity theorem for probability measures on compact groups (see, for example, Theorem 1.4.2 from [3]) that the measure $Q_{T}$ converges weakly to $\underline{m}_{3 H}$ as $T \rightarrow \infty$.

\section{Limit Theorems for Absolutely Convergent Series}

For fixed $\hat{\sigma}>\frac{1}{2}$, and $m \in \mathbb{N}_{0}, n \in \mathbb{N}$, let

$$
v\left(m, n, \alpha_{j}\right)=\exp \left\{-\left(\frac{m+\alpha_{j}}{n+\alpha_{j}}\right)^{\hat{\sigma}}\right\}, \quad j=1, \ldots, r .
$$

Define

$$
\zeta_{n}\left(s, \alpha_{j} ; \mathfrak{a}_{j}\right)=\sum_{m=0}^{\infty} \frac{a_{m j} v\left(m, n, \alpha_{j}\right)}{\left(m+\alpha_{j}\right)^{s}}, \quad j=1, \ldots, r .
$$

Then the series for $\zeta_{n}\left(s, \alpha_{j} ; \mathfrak{a}_{j}\right)$ converges absolutely for $\sigma>\frac{1}{2}$ independently on the arithmetical nature of $\alpha_{j}[4]$. For $A \in \mathcal{B}\left(\mathbb{C}^{r}\right)$, we set

$$
P_{T, n}(A)=\frac{1}{T} \operatorname{meas}\left\{t \in[0, T]: \underline{\zeta}_{n}(\underline{\sigma}+\mathrm{i} t, \underline{\alpha} ; \underline{\mathfrak{a}}) \in A\right\},
$$

where $\underline{\zeta}_{n}(\underline{\sigma}+\mathrm{i} t, \underline{\alpha} ; \underline{\mathfrak{a}})=\left(\zeta_{n}\left(\sigma_{1}+\mathrm{i} t, \alpha_{1} ; \mathfrak{a}_{1}\right), \ldots, \zeta_{n}\left(\sigma_{r}+\mathrm{i} t, \alpha_{r} ; \mathfrak{a}_{r}\right)\right)$. Moreover, for $\underline{\omega}_{3} \stackrel{\underline{n}}{=}\left(\omega_{31}, \ldots, \omega_{3 r}\right) \in \underline{\Omega}_{3}$, let

$$
\zeta_{n}\left(s, \alpha_{j}, \omega_{3 j} ; \mathfrak{a}_{j}\right)=\sum_{m=0}^{\infty} \frac{a_{m j} \omega_{3 j}(m) v\left(m, n, \alpha_{j}\right)}{\left(m+\alpha_{j}\right)^{s}}, \quad j=1, \ldots, r .
$$

Obviously, the series for $\zeta_{n}\left(s, \alpha_{j}, \omega_{3 j} ; \mathfrak{a}_{j}\right)$ also converges absolutely for $\sigma>\frac{1}{2}$. Let $\underline{\zeta}_{n}\left(\underline{\sigma}+\mathrm{i} t, \underline{\alpha}, \underline{\omega}_{3} ; \underline{\mathfrak{a}}\right)=\left(\zeta_{n}\left(\sigma_{1}+\mathrm{i} t, \alpha_{1}, \underline{\omega}_{31} ; \mathfrak{a}_{1}\right), \ldots, \zeta_{n}\left(\sigma_{r}+\mathrm{i} t, \alpha_{r}, \omega_{3 r} ; \mathfrak{a}_{r}\right)\right)$, and, for $A \in \mathcal{B}\left(\mathbb{C}^{r}\right)$ and a fixed $\underline{\hat{\omega}}_{3}=\left(\hat{\omega}_{31}, \ldots, \hat{\omega}_{3 r}\right)$,

$$
\hat{P}_{T, n}(A)=\frac{1}{T} \operatorname{meas}\left\{t \in[0, T]: \underline{\zeta}_{n}\left(\underline{\sigma}+\mathrm{i} t, \underline{\alpha}, \underline{\hat{\omega}}_{3} ; \underline{\mathfrak{a}}\right) \in A\right\} .
$$

Theorem 5. Suppose that $\min _{1 \leq j \leq r} \sigma_{j}>\frac{1}{2}$. Then, on $\left(\mathbb{C}^{r}, \mathcal{B}\left(\mathbb{C}^{r}\right)\right)$, there exists a probability measure $P_{n}$ such that the measures $P_{T, n}$ and $\hat{P}_{T, n}$ both converge weakly to $P_{n}$ as $T \rightarrow \infty$.

Proof. Define a function $h_{n}: \underline{\Omega}_{3} \rightarrow \mathbb{C}^{r}$ by the formula

$$
h_{n}\left(\underline{\omega}_{3}\right)=\left(\sum_{m=0}^{\infty} \frac{a_{m 1} \omega_{31}(m) v\left(m, n, \alpha_{1}\right)}{\left(m+\alpha_{1}\right)^{\sigma_{1}}}, \ldots, \sum_{m=0}^{\infty} \frac{a_{m r} \omega_{3 r}(m) v\left(m, n, \alpha_{r}\right)}{\left(m+\alpha_{r}\right)^{\sigma_{r}}}\right) .
$$

Since the series in the definition of $h_{n}$ converge absolutely, the function $h_{n}$ is continuous, moreover,

$$
\begin{aligned}
& h_{n}\left(\left(\left(m+\alpha_{1}\right)^{-\mathrm{i} t}: m \in \mathcal{M}\left(\alpha_{1}\right)\right), \ldots,\left(\left(m+\alpha_{r}\right)^{-\mathrm{i} t}: m \in \mathcal{M}\left(\alpha_{r}\right)\right)\right) \\
& \quad=\left(\zeta_{n}\left(\sigma_{1}+\mathrm{i} t, \alpha_{1} ; \mathfrak{a}_{1}\right), \ldots, \zeta_{n}\left(\sigma_{r}+\mathrm{i} t, \alpha_{r} ; \mathfrak{a}_{r}\right)\right)=\underline{\zeta}_{n}(\underline{\sigma}+\mathrm{i} t, \underline{\alpha} ; \underline{\mathfrak{a}}) .
\end{aligned}
$$


Hence, we have that

$$
P_{T, n}(A)=Q_{T} h_{n}^{-1}(A), \quad A \in \mathcal{B}\left(\mathbb{C}^{r}\right) .
$$

Therefore, Theorem 4 together with Theorem 5.1 from [1] show that the measure $P_{T, n}$ converges weakly to $\underline{m}_{3 H} h_{n}^{-1}$ as $T \rightarrow \infty$.

It remains to prove that the measure $\hat{P}_{T, n}$ also converges weakly to $\underline{m}_{3 H} h_{n}^{-1}$ as $T \rightarrow \infty$. Let a function $h: \underline{\Omega}_{3} \rightarrow \underline{\Omega}_{3}$ be given by the formula $h\left(\underline{\omega}_{3}\right)=\underline{\omega}_{3} \hat{\omega}_{3}$. Then we have that

$$
\begin{aligned}
h_{n} & \left(h\left(\left(\left(\left(m+\alpha_{1}\right)^{-\mathrm{i} t}: m \in \mathcal{M}\left(\alpha_{1}\right)\right), \ldots,\left(\left(m+\alpha_{r}\right)^{-\mathrm{i} t}: m \in \mathcal{M}\left(\alpha_{r}\right)\right)\right)\right)\right) \\
& =\left(\zeta_{n}\left(\sigma_{1}+\mathrm{i} t, \alpha_{1}, \omega_{31} ; \mathfrak{a}_{1}\right), \ldots, \zeta_{n}\left(\underline{\sigma}_{r}+\mathrm{i} t, \alpha_{r}, \omega_{3 r} ; \mathfrak{a}_{r}\right)\right) \\
& =\underline{\zeta}_{n}\left(\underline{\sigma}+\mathrm{i} t, \underline{\alpha}, \underline{\hat{\omega}}_{3} ; \underline{\mathfrak{a}}\right) .
\end{aligned}
$$

Thus, the above arguments show that the measure $\hat{P}_{T, n}$ converges weakly to $\underline{m}_{3 H}\left(h_{n} h\right)^{-1}$ as $T \rightarrow \infty$. However, the invariance of the Haar measure $\underline{m}_{3 H}$ implies the equality $\underline{m}_{3 H}\left(h_{n} h\right)^{-1}=\left(\underline{m}_{3 H} h^{-1}\right) h_{n}^{-1}=\underline{m}_{3 H} h_{n}^{-1}$.

\section{Approximation in the Mean}

In this section, we approximate $\underline{\zeta}(\underline{\sigma}+\mathrm{i} t, \underline{\alpha}, ; \underline{\mathfrak{a}})$ by $\underline{\zeta}_{n}(\underline{\sigma}+\mathrm{i} t, \underline{\alpha}, ; \underline{\mathfrak{a}})$, and $\underline{\zeta}(\underline{\sigma}+$ it $\left., \underline{\alpha}, \underline{\omega}_{3} ; \underline{\mathfrak{a}}\right)$ by $\underline{\zeta}_{n}\left(\underline{\sigma}+\mathrm{i} t, \underline{\alpha}, \underline{\omega}_{3} ; \underline{\mathfrak{a}}\right)$. We use the Euclidean metric $\rho$ in $\mathbb{C}^{r}$. Let $\underline{z}_{j}=\left(z_{j 1}, \ldots, z_{j r}\right) \in \mathbb{C}^{r}, j=1,2$, and

$$
\rho\left(\underline{z}_{1}, \underline{z}_{2}\right)=\left(\sum_{j=1}^{r}\left|z_{1 j}-z_{2 j}\right|^{2}\right)^{\frac{1}{2}}
$$

Lemma 1. Suppose that $\min _{1 \leq j \leq r} \sigma_{j}>\frac{1}{2}$. Then

$$
\lim _{n \rightarrow \infty} \limsup _{T \rightarrow \infty} \frac{1}{T} \int_{0}^{T} \rho\left(\underline{\zeta}(\underline{\sigma}+\mathrm{i} t, \underline{\alpha}, ; \underline{\mathfrak{a}}), \underline{\zeta}_{n}(\underline{\sigma}+\mathrm{i} t, \underline{\alpha}, ; \underline{\mathfrak{a}})\right) \mathrm{d} t=0 .
$$

Proof. By Lemma 6 from [6], we have that, for every $j=1, \ldots, r$,

$$
\lim _{n \rightarrow \infty} \limsup _{T \rightarrow \infty} \frac{1}{T} \int_{0}^{T}\left|\zeta\left(\sigma_{j}+\mathrm{i} t, \alpha_{j} ; \mathfrak{a}_{j}\right)-\zeta_{n}\left(\sigma_{j}+\mathrm{i} t, \alpha_{j}, ; \mathfrak{a}_{j}\right)\right| \mathrm{d} t=0 .
$$

Since

$$
\rho\left(\underline{z}_{1}, \underline{z}_{2}\right) \leq \sum_{j=1}^{r}\left|z_{1 j}-z_{2 j}\right|,
$$

this proves the lemma.

Lemma 2. Suppose that $\min _{1 \leq j \leq r} \sigma_{j}>\frac{1}{2}$. Then, for almost all $\underline{\omega}_{3} \in \underline{\Omega}_{3}$,

$$
\lim _{n \rightarrow \infty} \limsup _{T \rightarrow \infty} \frac{1}{T} \int_{0}^{T} \rho\left(\underline{\zeta}\left(\underline{\sigma}+\mathrm{i} t, \underline{\alpha}, \underline{\omega}_{3} ; \underline{\mathfrak{a}}\right), \underline{\zeta}_{n}\left(\underline{\sigma}+\mathrm{i} t, \underline{\alpha}, \underline{\omega}_{3} ; \underline{\mathfrak{a}}\right)\right) \mathrm{d} t=0 \text {. }
$$


Proof. By Lemma 15 from [6], for almost all $\omega_{3 j} \in \Omega_{3 j}$ and every $j=1, \ldots, r$,

$$
\lim _{n \rightarrow \infty} \limsup _{T \rightarrow \infty} \frac{1}{T} \int_{0}^{T}\left|\zeta\left(\sigma_{j}+\mathrm{i} t, \alpha_{j}, \omega_{3 j} ; \mathfrak{a}_{j}\right)-\zeta_{n}\left(\sigma_{j}+\mathrm{i} t, \alpha_{j}, \omega_{3 j} ; \mathfrak{a}_{j}\right)\right| \mathrm{d} t=0
$$

Let $\hat{\Omega}_{3 j}$ be the subset of $\Omega_{3 j}$ whose elements satisfy the later relation. Then we have that $m_{3 j H}\left(\hat{\Omega}_{3 j}\right)=1$, where $m_{3 j H}$ is the Haar measure on $\left(\Omega_{3 j}, \mathcal{B}\left(\Omega_{3 j}\right)\right)$, $j=1, \ldots, r$. Let $\underline{\Omega}_{3}=\hat{\Omega}_{31} \times \cdots \times \hat{\Omega}_{3 r}$. Since the Haar measure $\underline{m}_{3 H}$ is the product of the measures $m_{31 H}, \ldots, m_{3 r H}$, we find that $\underline{m}_{3 H}\left(\underline{\hat{\Omega}}_{3}\right)=1$. Therefore, the assertion of the lemma follows from inequality (4.1) and relation (4.2).

\section{Limit Theorems for $\underline{\zeta}(s, \underline{\alpha} ; \underline{\mathfrak{a}})$ and $\underline{\zeta}_{3}\left(s, \underline{\alpha}, \underline{\omega}_{3} ; \underline{\mathfrak{a}}\right)$}

For $A \in \mathcal{B}\left(\mathbb{C}^{r}\right)$, define one more probability measure

$$
\hat{P}_{T}(A)=\frac{1}{T} \operatorname{meas}\left\{t \in[0, T]: \underline{\zeta}_{3}\left(\underline{\sigma}+\mathrm{i} t, \underline{\alpha}, \underline{\omega}_{3} ; \underline{\mathfrak{a}}\right) \in A\right\} .
$$

Theorem 6. Suppose that the numbers $\alpha_{1}, \ldots, \alpha_{r}$ satisfy the hypotheses of Theorem 3 , and that $\min _{1 \leq j \leq r} \sigma_{j}>\frac{1}{2}$. Then, on $\left(\mathbb{C}^{r}, \mathcal{B}\left(\mathbb{C}^{r}\right)\right)$, there exists a probability measure $P$ such that the measures $P_{T}$ and $\hat{P}_{T}$ both converge weakly to $P$ as $T \rightarrow \infty$.

Proof. Let $\theta$ be a random variable defined on a certain probability space $(\Omega, \mathcal{A}, \mathbb{P})$ and uniformly distributed on the interval $[0,1]$. Define

$$
\underline{X}_{T, n}=\underline{X}_{T, n}(\underline{\sigma})=\left(X_{T, n, 1}\left(\sigma_{1}\right), \ldots, X_{T, n, r}\left(\sigma_{r}\right)\right)=\underline{\zeta}(\underline{\sigma}+\mathrm{i} T \theta, \underline{\alpha} ; \underline{\mathfrak{a}})
$$

which is a $\mathbb{C}^{r}$-valued random element on the probability space $(\Omega, \mathcal{A}, \mathbb{P})$. Then we have, in view of Theorem 5 , that

$$
\underline{X}_{T, n} \underset{T \rightarrow \infty}{\stackrel{\mathcal{D}}{\longrightarrow}} \underline{X}_{n},
$$

where $\underline{X}_{n}=\underline{X}_{n}(\underline{\sigma})=\left(X_{n, 1}\left(\sigma_{1}\right), \ldots, X_{n, r}\left(\sigma_{r}\right)\right)$ is the $\mathbb{C}^{r}$-valued random element which distribution is the limit measure $P_{n}$ in Theorem 5, and, as usual, $\stackrel{\mathcal{D}}{\longrightarrow}$ means the convergence in distribution.

Since the series for $\zeta_{n}\left(\sigma_{j}+\mathrm{i} t, \alpha_{j} ; \mathfrak{a}_{j}\right)$ converges absolutely, the properties of Dirichlet series imply

$$
\lim _{T \rightarrow \infty} \frac{1}{T} \int_{0}^{T}\left|\zeta_{n}\left(\sigma_{j}+\mathrm{i} t, \alpha_{j} ; \mathfrak{a}_{j}\right)\right|^{2} \mathrm{~d} t=\sum_{m=0}^{\infty} \frac{\left|a_{m j}\right|^{2} v^{2}\left(m, n, \alpha_{j}\right)}{\left(m+\alpha_{j}\right)^{2 \sigma_{j}}} \leq \sum_{m=0}^{\infty} \frac{\left|a_{m j}\right|^{2}}{\left(m+\alpha_{j}\right)^{2 \sigma_{j}}},
$$

for all $n \in \mathbb{N}, j=1, \ldots, r$. Hence, for $j=1, \ldots, r$,

$$
\limsup _{T \rightarrow \infty} \frac{1}{T} \int_{0}^{T}\left|\zeta_{n}\left(\sigma_{j}+\mathrm{i} t, \alpha_{j} ; \mathfrak{a}_{j}\right)\right| \mathrm{d} t \leq R_{j}
$$


where $R_{j}=\left(\sum_{m=0}^{\infty} \frac{\left|a_{m j}\right|^{2}}{\left(m+\alpha_{j}\right)^{2 \sigma_{j}}}\right)^{\frac{1}{2}}<\infty$. Now let $\varepsilon>0$ be arbitrary number, and $M_{j}=M_{j}(\varepsilon)=R_{j} r \varepsilon^{-1}, j=1, \ldots, r$. Then, in view of (5.2),

$$
\begin{aligned}
& \limsup _{T \rightarrow \infty} \mathbb{P}\left(\left|X_{T, n, j}\left(\sigma_{j}\right)\right|>M_{j} \text { for at least one } j=1, \ldots, r\right) \\
& \quad \leq \sum_{j=1}^{r} \limsup _{T \rightarrow \infty} \mathbb{P}\left(\left|X_{T, n, j}\left(\sigma_{j}\right)\right|>M_{j}\right) \\
& \quad \leq \sum_{j=1}^{r} \limsup _{T \rightarrow \infty} \frac{1}{T} \operatorname{meas}\left\{t \in[0, T]:\left|\zeta_{n}\left(\sigma_{j}+\mathrm{i} t, \alpha_{j} ; \mathfrak{a}_{j}\right)\right| \geq M_{j}\right\} \\
& \quad \leq \sum_{j=1}^{r} \frac{1}{M_{j}} \sup _{n \in \mathbb{N}} \limsup _{T \rightarrow \infty} \frac{1}{T} \int_{0}^{T}\left|\zeta_{n}\left(\sigma_{j}+\mathrm{i} t, \alpha_{j} ; \mathfrak{a}_{j}\right)\right| \mathrm{d} t \leq \sum_{j=1}^{r} R_{j} / M_{j}=\varepsilon .
\end{aligned}
$$

This together with (5.1) implies

$$
\mathbb{P}\left(\left|X_{n, j}\left(\sigma_{j}\right)\right|>M_{j} \text { for at least one } j=1, \ldots, r\right) \leq \varepsilon .
$$

for all $n \in \mathbb{N}$. Now define $K_{\varepsilon}^{r}=\left\{\underline{z} \in \mathbb{C}^{r}:\left|z_{j}\right| \leq M_{j}, j=1, \ldots, r\right\}$. Then $K_{\varepsilon}$ is a bounded closed set, thus it is a compact set on $\mathbb{C}^{r}$. Moreover, by (5.3),

$$
\mathbb{P}\left(\underline{X}_{n} \in K_{\varepsilon}^{r}\right) \geq 1-\varepsilon,
$$

or, by the definition of $\underline{X}_{n}$,

$$
P_{n}\left(K_{\varepsilon}^{r}\right) \geq 1-\varepsilon
$$

for all $n \in \mathbb{N}$. This means that the family of probability measures $\left\{P_{n}: n \in \mathbb{N}\right\}$ is tight, and, by the Prokhorov theorem, see, for example, [1], it is relatively compact. Therefore, there exists a subsequence $\left\{P_{n_{k}}\right\} \subset\left\{P_{n}\right\}$ such that the measure $P_{n_{k}}$ converges weakly to a certain probability measure $P$ on $\left(\mathbb{C}^{r}, \mathcal{B}\left(\mathbb{C}^{r}\right)\right)$ as $k \rightarrow \infty$. In other words,

$$
\underline{X}_{n_{k}} \underset{k \rightarrow \infty}{\stackrel{\mathcal{D}}{\longrightarrow}} P \text {. }
$$

Now define

$$
\underline{X}_{T}=\underline{X}_{T}(\underline{\sigma})=\underline{\zeta}(\underline{\sigma}+\mathrm{i} T \theta, \underline{\alpha} ; \underline{\mathfrak{a}}) .
$$

Then $\underline{X}_{T}$ is a $\mathbb{C}^{r}$-valued random element on the probability space $(\Omega, \mathcal{A}, \mathbb{P})$. Using Lemma 1.1, we find that, for every $\varepsilon>0$,

$$
\begin{aligned}
& \lim _{n \rightarrow \infty} \limsup _{T \rightarrow \infty} \frac{1}{T} \operatorname{meas}\left\{t \in[0, T]: \rho\left(\underline{\zeta}(\underline{\sigma}+\mathrm{i} t, \underline{\alpha} ; \underline{\mathfrak{a}}), \underline{\zeta}_{n}(\underline{\sigma}+\mathrm{i} t, \underline{\alpha} ; \underline{\mathfrak{a}})\right) \geq \varepsilon\right\} \\
& \quad \leq \lim _{n \rightarrow \infty} \limsup _{T \rightarrow \infty} \frac{1}{\varepsilon T} \int_{0}^{T} \rho\left(\underline{\zeta}(\underline{\sigma}+\mathrm{i} t, \underline{\alpha} ; \underline{\mathfrak{a}}), \underline{\zeta}_{n}(\underline{\sigma}+\mathrm{i} t, \underline{\alpha} ; \underline{\mathfrak{a}})\right) \mathrm{d} t=0 .
\end{aligned}
$$

This and the definitions of the random elements $\underline{X}_{T, n}$ and $\underline{X}_{T}$ imply

$$
\lim _{n \rightarrow \infty} \limsup _{T \rightarrow \infty} \mathbb{P}\left(\rho\left(\underline{X}_{T}, \underline{X}_{T, n}\right) \geq \varepsilon\right)=0 .
$$


The relations (5.1), (5.4) and (5.5) show that the hypotheses of Theorem 4.2 from [1] are satisfied. Therefore,

$$
\underline{X}_{T} \underset{T \rightarrow \infty}{\stackrel{\mathcal{D}}{\longrightarrow}} P
$$

and this shows that the measure $P_{T}$ converges weakly to $P$ as $T \rightarrow \infty$. Moreover, in virtue of (5.6) we have that the measure $P$ is independent of the choice of the subsequence $P_{n_{k}}$. Thus, the relative compactness of the family $\left\{P_{n}\right\}$ yields the relation

$$
\underline{X}_{n} \underset{n \rightarrow \infty}{\stackrel{\mathcal{D}}{\longrightarrow}} P \text {. }
$$

Reasoning similarly to the case of the measure $P_{T}$ and using Theorem 5 , Lemma 2 and (5.7), we obtain without difficulty that the measure $\hat{P}_{T}$ also converges weakly to $P$ as $T \rightarrow \infty$.

In view of Theorem 6 , for proving Theorem 3 , it remains to identify the measure $P$ in (5.6).

\section{Proof of Theorem 3}

We start with one statement from ergodic theory. Let $\underline{a}_{t}=\left\{\left(\left(m+\alpha_{1}\right)^{-\mathrm{i} t}: m \in\right.\right.$ $\left.\left.\mathcal{M}\left(\alpha_{1}\right)\right), \ldots,\left(\left(m+\alpha_{r}\right)^{-\mathrm{i} t}: m \in \mathcal{M}\left(\alpha_{r}\right)\right)\right\}, t \in \mathbb{R}$. Define the one-parameter family $\left\{\Phi_{t}: t \in \mathbb{R}\right\}$ of transformation of $\underline{\Omega}_{3}$ by the formula $\Phi_{t}\left(\underline{\omega}_{3}\right)=\underline{a}_{t} \underline{\omega}_{3}$, $\underline{\omega}_{3} \in \underline{\Omega}_{3}$. Then $\left\{\Phi_{t}: t \in \mathbb{R}\right\}$ is a one-parameter group of measurable measure preserving transformations on the group $\underline{\Omega}_{3}$.

Lemma 3. Suppose that the numbers $\alpha_{1}, \ldots, \alpha_{r}$ satisfy the hypotheses of Theorem 3 . Then the one-parameter group $\left\{\Phi_{t}: t \in \mathbb{R}\right\}$ is ergodic.

Proof. Let $\chi$ be a character of the group $\underline{\Omega}_{3}$. We have seen in the proof of Theorem 4 that

$$
\chi\left(\underline{\omega}_{3}\right)=\prod_{m \in \mathcal{M}\left(\alpha_{1}\right)} \omega_{31}^{k_{1 m}}(m) \ldots \prod_{m \in \mathcal{M}\left(\alpha_{r}\right)} \omega_{3 r}^{k_{r m}}(m), \quad \underline{\omega}_{3}=\left(\omega_{31}, \ldots, \omega_{3 r}\right) \in \underline{\Omega}_{3},
$$

where only a finite number of integers $k_{j m}, j=1, \ldots, r$, are distinct from zero. First suppose that $\chi$ is a non-trivial character. Then we have that

$$
\begin{aligned}
& \chi\left(\underline{a}_{t}\right)=\prod_{m \in \mathcal{M}\left(\alpha_{1}\right)}\left(m+\alpha_{1}\right)^{-\mathrm{i} t k_{1 m}} \cdots \prod_{m \in \mathcal{M}\left(\alpha_{r}\right)}\left(m+\alpha_{r}\right)^{-\mathrm{i} t k_{r m}} \\
& =\exp \left\{-\mathrm{i} t\left(\sum_{m \in \mathcal{M}\left(\alpha_{1}\right)} k_{1 m} \log \left(m+\alpha_{1}\right)+\cdots+\sum_{m \in \mathcal{M}\left(\alpha_{r}\right)} k_{r m} \log \left(m+\alpha_{r}\right)\right)\right\} .
\end{aligned}
$$

Using the linear independence of the set $\bigcup_{j=1}^{r} I\left(\alpha_{j}\right)$, hence, we find that there exists $\tau_{0} \in \mathbb{R} \backslash\{0\}$ such that $\chi\left(\underline{a}_{\tau_{0}}\right)=1$. The further proof is standard, see, for example, [5]. 
Proof of Theorem 3. We take a fixed continuity set $A$ of the limit measure $P$ in Theorem 6 . Then, using the equivalent of the weak convergence of probability measures in terms of continuity sets, see Theorem 2.1 from [1], we have that

$$
\lim _{T \rightarrow \infty} \frac{1}{T} \operatorname{meas}\left\{t \in[0, T]: \underline{\zeta}_{3}\left(\underline{\sigma}+\mathrm{i} t, \underline{\alpha}, \underline{\omega}_{3} ; \underline{\mathfrak{a}}\right) \in A\right\}=P(A) .
$$

Let $\hat{\theta}$ be a random variable on $\left(\underline{\Omega}_{3}, \mathcal{B}\left(\underline{\Omega}_{3}\right), \underline{m}_{3 H}\right)$ given by

$$
\hat{\theta}\left(\underline{\omega}_{3}\right)= \begin{cases}1 & \text { if } \underline{\zeta}_{3}\left(\underline{\sigma}, \underline{\alpha}, \underline{\omega}_{3} ; \underline{\mathfrak{a}}\right) \in A, \\ 0 & \text { otherwise. }\end{cases}
$$

Then, obviously, the expectation $\mathbb{E}(\hat{\theta})$ of $\hat{\theta}$ equals

$$
\int_{\underline{\Omega}_{3}} \hat{\theta} \mathrm{d} \underline{m}_{3 H}=\underline{m}_{3 H}\left(\underline{\omega}_{3} \in \underline{\Omega}_{3}: \underline{\zeta}_{3}\left(\underline{\sigma}, \underline{\alpha}, \underline{\omega}_{3} ; \underline{\mathfrak{a}}\right) \in A\right)=P_{\underline{\zeta}_{3}}(A) .
$$

From Lemma 3 , the ergodicity of the random process $\hat{\theta}\left(\Phi_{t}\left(\underline{\omega}_{3}\right)\right)$ follows. Therefore, an application of the classical Birkhoff-Khintchine theorem, see, for example, [2], shows that, for almost all $\underline{\omega}_{3} \in \underline{\Omega}_{3}$,

$$
\lim _{T \rightarrow \infty} \frac{1}{T} \int_{0}^{T} \hat{\theta}\left(\Phi_{t}\left(\underline{\omega}_{3}\right)\right) \mathrm{d} t=\mathbb{E}(\hat{\theta}) .
$$

On the other hand, by the definitions of $\hat{\theta}$ and $\Phi_{t}$, we have that

$$
\frac{1}{T} \int_{0}^{T} \hat{\theta}\left(\Phi_{t}\left(\underline{\omega}_{3}\right)\right) \mathrm{d} t=\frac{1}{T} \operatorname{meas}\left\{t \in[0, T]: \underline{\zeta}_{3}\left(\underline{\sigma}+\mathrm{i} t, \underline{\alpha}, \underline{\omega}_{3} ; \underline{\mathfrak{a}}\right) \in A\right\} .
$$

This, (6.2) and (6.3) imply, for almost all $\underline{\omega}_{3} \in \underline{\Omega}_{3}$, the equality

$$
\lim _{T \rightarrow \infty} \frac{1}{T} \operatorname{meas}\left\{t \in[0, T]: \underline{\zeta}_{3}\left(\underline{\sigma}+\mathrm{i} t, \underline{\alpha}, \underline{\omega}_{3} ; \underline{\mathfrak{a}}\right) \in A\right\}=P_{\underline{\zeta}_{3}}(A) .
$$

Hence, taking into account (6.1), we obtain that $P(A)=P_{\underline{\zeta}_{3}}(A)$. Since $A$ was an arbitrary continuity set of $P$, the latter relation is true for all continuity sets of $P$, and this shows that $P(A)=P_{\underline{\zeta}_{3}}(A)$ for all $A \in \mathcal{B}\left(\mathbb{C}^{r}\right)$.

\section{References}

[1] P. Billingsley. Convergence of Probability Measures. Wiley, New York, 1968.

[2] H. Cramér and M.R. Leadbetter. Stationary and Related Stochastic Processes. Wiley, New York, 1967.

[3] H. Heyer. Probability Measures on Locally Compact Groups. Springer-Verlag, Berlin, 1977.

[4] A. Javtokas and A. Laurinčikas. On the periodic Hurwitz zeta-function. HardyRamanujan J., 29(3):18-36, 2006.

[5] A. Laurinčikas and R. Garunkštis. The Lerch Zeta-Function. Kluwer, Dordrecht, Boston, London, 2002.

[6] A. Rimkevičienè. Limit theorems for the periodic Hurwitz zeta-function. Šiauliai Math. Semin., 5(13):55-69, 2010.

[7] A. Rimkevičienè. Joint limit theorems for periodic Hurwitz zeta-functions. Šiauliai Math. Semin., 6(14):53-68, 2011. 